\title{
““Ideal” approaches to Cancer drug design
}

\section{A REVIEW}

\author{
Pardis Tabaee Damavandi
}

\begin{abstract}
Author Note
Copyright:@ the author(s), publisher and licensee.This is an open-access article distributed under the terms of the Creative Commons Attribution Non-Commercial License, which permits unrestricted non-commercial use, distribution, and reproduction in any medium, provided the original work is properly cited.
\end{abstract}




\begin{abstract}
In this review we present potential approaches to drug design that can facilitate cancer treatment, which so far has been difficult to achieve in the safest manner, due to the complexity of disease, presented with multiple mutations. Targeting mutant proteins is not simple as mutations are plural and sometimes unmanageable due to their large number. Many therapies have failed as a result, although we have good prognosis for the majority of cancers nowadays, it is important to understand what can be done in late stage disease.

Keywords: Cancer, Drug Design, Pharmacology, Chemotherapy, Telomerase, Co-inhibitors, Prodrugs
\end{abstract}




\section{"Ideal" approaches to Cancer drug design}

\section{A REVIEW}

Cancer drug design is very important and possibly the most intriguing yet "nerve-racking" uncertainty current pharmacologists have to face due to the plurality of problems the organism goes through whilst succumbing to the disease. Even though chemotherapy is successful and has led to complete healing of even the most aggressive cancers, it remains the second leading cause of death in the world ${ }^{1}$.

Classical approaches to drug development in the oncology context have been based on targeting cell growth, which as we know, is rapid in most cancers (unlike in healthy cells), particularly the blood forms, but have also included gene therapy, radiotherapy, invasive methods in blood cancers, blockages of metastasis and angiogenesis, chemotherapy and stem cell technology and supplementation procedures. All these treatments that are performed sometimes lead to remissions, but often to complete healing, yet, these harsh approaches have important side effects and do not always stop the disease, nor ameliorate it to the extent that we expect them to, because the drugs are very a-specific. Clearly, drugs that target metastasis and angiogenesis ${ }^{2}$ are selective, but most chemotherapeutic agents act directly at nucleic acid level or are monoclonal antibodies.

Chemotherapeutic agents are a wonderful option, but what if we could improve drug design and obtain an even better outcome? The potential of finding a cure rather than just treating the disease has become more of interest recently thanks to particular discoveries that I am going to elucidate further. 


\section{Multiple mutations versus single mutations}

The primary challenge that we observe in the pathology of cancer as opposed to other diseases is the amount of mutations that lead to the disease. When we commonly speak about cancer we are never dealing with one single or a few mutations, we are not talking about $\mathrm{n}<10$ mutations, but quite often a large number of mutations per gene, and per each gene we often find that the amount of mutated proteins involved that are synthesized by those same genes are even higher in number. This makes targeting of the mutations very difficult. It is not difficult when we target metastasis; for example, inhibiting neuropilin-1 which is one of the proteins involved in metastasis has proven to be beneficial, because this approach is very "focused" and "zoomed-in" onto the process of metastasis prophylaxis, but it won't stop the tumor from continuous growth. The tumour remains benign, may grow larger, but it still requires surgical removal, as it becomes cumbersome to the nearby organs; this is because tumoural growth has not been stopped, simply, its metastasis has been "obstacled" to prevent it from invading other organic compartments and becoming malignant. The therapy is successful, because the targeting of proteins involved in angiogenesis is enough, not allowing the tumour to metastasise further, but the accumulation of the already metastatic cells needs treatment too, leading to cocktails of chemotherapeutic agents and thus to the polypharmacy we experience in the clinical settings.

Nonetheless, many researchers have been attempting the targeting of each mutation that

they find associated with a particular cancer, however, literature on the matter is perplexing and difficult to understand, sometimes even contradictory, and scattered, as each group privileges one mutation over the other, even though many mutations are involved. It is an understandably desperate approach to cancer, particularly for late stage cancers that have escaped scrutiny and metastised. Evidently anti-metastatic drugs are used in cancer clinical trials nevertheless for 
preventing the worsening of the disease, but they do not remove already malignant cells circulating in the blood. Not only is this a challenge, but also the lack of knowledge about the source of certain cancers, which in the literature have been associated to multiple causes, hinders research. Exemplary, are the myeloblasts in Acute Myeloid Leukemia (AML), where myeloblasts do not maturate into differentiated corpuscular elements of the blood inducing anemia and other complications, but the causes to this devolution are often retained idiopathic. There exist many hypotheses on the source of several types of cancers, comprising discussions about potential or mineral-vitamin deficiencies, cancers that may be secondary to certain drugs (drug misuse) and diseases, exposure to chemicals such as aromatic compounds (benzene), and more specifically an exposure to a combination of at least two of these hazardous substances, genetic susceptibility and so on, which make predisposition to cancer likely, but drug development difficult. Microscopic evidence has however, emerged throughout the years indicating that mutations are common denominators for all cancers; this is also true for the majority of diseases, but the main difference between cancers and other diseases is the above-mentioned amount of mutations, which is dire in the case of cancer, making the targeting of those mutations difficult, and thus leading to certain radical approaches such as gene therapy (or gene replacement technology). Scientists have lately endeavored prioritizing the mapping of mutations in what is a "cascade of mutations", however, more recent studies have shown that mutations are linked to the shortening of telomeres associated with aging ${ }^{3}$; this means that telomere shortening is the source of mutations that we observe in cancer and even in other diseases, because the genetic material that composes our cells becomes "old" and that this shortening is inevitable as we age, leading to disease. Though, cancers can be found in the youngest population also proven to express short telomeres, yet, in the youth, healing 
occurs with standard chemotherapy often in a percentage higher than $92 \%$ of the people without relapse.

This is because it is the telomerase enzyme which is the culprit for telomere shortening, to begin with, and more specifically its idiopathic mutations; below, potential approaches to drug design are therefore discussed.

\section{Designing mutant telomerase inhibitors}

One way to solve telomerase mutation to prevent cancer is to design drugs that target the mutations of polymorphic telomerases associated with disease, instead of targeting the wild type form. This can be achieved with both classical and novel drug design approaches. Human telomerase contains approximately 1132 amino acids $^{4}$ and polymorphisms of the protein have been associated unexclusively with a large variety of cancers. This strategy could benefit particularly blood cancers.

\section{Co-inhibitor prodrugs}

Co-inhibitor drugs have existed historically, famously in the case of Augmentin ${ }^{\circledR}$ whereby two inhibitor molecules had a synergistic action with one operating as a masking, "tricking" agent, in the form of Clavulanic Acid, together with the actual antibiotic sister compound, Amoxicillin, however, theirs, was an association of two "independent" drugs; the combinatory success is "heavily" established by its formulation and drug delivery optimisations, aside from its clever medicinal chemistry. The strategy hereby proposed is to use drug design approaches to study the several mutations involved specifically in each cancer, and to then design one single entity as a drug, but "ramified" into "prodrugs" that are released once in the organism. To exemplify, if we were considering an aggressive life-threatening type of cancer, which theoretically involved two mutated genes "translating" into hypothetically four enzymatic relevant mutations, then the design of selective drugs for each mutation "bridged" together as prodrugs could be exploited. This 
strategy could help improve selectivity thus diminishing side effects, as it would lead to the clearance of the "sick" proteins (bound to the "liberated" drugs). Metabolic toxicity could potentially involve the kidneys due to the large amount of protein eliminated, but kidney monitoring or controlled drug delivery strategies may help refining this aspect too.

\section{Conclusion}

Drug design of novel cancer agents could involve the targeting of "sick" mutant telomerases which are easier to manipulate than targeting multiple mutations involved in a single cancer, because the number of mutated proteins or genes is extremely elevated in this pathology, unlike in other diseases, where receptor-drug binding is much easier. Nonetheless if we were to handle several mutations at once rather than designing n number of drugs separately, we could design one single drug in the form of a "pluri-prodrug" targeting several mutations that can be released all at once, for the specific cancer at hand. This strategy could be an alternative to aggressive chemotherapy as it would enhance selectivity leading to reduced adverse effects making therapy safer, more effective, and qualitative.

No conflicts of interest to disclose 


\section{References}

1. Ferlay J., Ervik M., Lam F. et al., Global Cancer Observatory: Cancer Today. Lyon: International Agency for Research on Cancer; 2020 (https://gco.iarc.fr/today).

2. Tabaee Damavandi P., Tuttle C. T., (2011), Computational studies of ATWLPPR and analogues as Neuropilin-1 inhibitors", United Kingdom.

3. Blackburn EH, Greider CW, Szostak JW . (2006). Telomeres and telomerase: the path from maize, Tetrahymena and yeast to human cancer and aging. Nat Med 12: 11331138.

4. Cohen S. B., Graham M. E., Lovrecz G. O. et al. (2007), Protein Composition of Catalytically Active Human Telomerase from Immortal Cells, Science, 315(5820): $1850-1853$. 